Mélodie Chauret Université de Sherbrooke

Isabelle Carignan

Université TÉLUQ

Vincent Grenon

Université de Sherbrooke

Simon Collin

Université du Québec à Montréal

\section{Les compétences informationnelles d'enseignants du primaire et du secondaire lors d'une recherche par mots-clés sur un moteur de recherche}

\author{
The information literacy skills of elementary and high school \\ teachers during a search by keywords on a search engine
}

\section{ésumé}

Étant donné l'importance de savoir chercher, évaluer et utiliser les informations sur Internet, cette étude vise à connaître les compétences informationnelles utilisées par les enseignants afin de documenter, par observation, leur mode de fonctionnement et leur niveau d'aisance lors $d^{\prime}$ une recherche par mots-clés. Cette recherche descriptive exploratoire a permis la découverte de nouvelles stratégies de recherche ainsi que I'adaptation et l'enrichissement d'un modèle de compétences informationnelles développé à partir de modèles existants. En conclusion, nous analysons ces résultats par rapport à la recherche existante tout en offrant des pistes de recommandations en vue de bonifier la formation des enseignants.

Mots-clés

Compétences informationnelles, stratégies de recherche, lecture à l'écran, modélisation.

Abstract

Given the importance of knowing how to search, evaluate and use information on the Internet, this study aims to know the informational skills used by teachers in order to document, by observation, their mode of operation and their level of ease during a keyword research. This descriptive and exploratory research allowed the discovery of new research strategies as well as the adaptation and enrichment of an information skills model developed from existing models. In conclusion, we analyze these results in relation

to existing research while offering avenues of recommendations for improving teacher training.

Keywords

Information literacy skills; information search strategies; reading online; modelization.

\section{Introduction}

L'utilisation d'Internet fait de plus en plus partie du contexte de la classe. Les élèves, que ce soit au primaire ou au secondaire, utilisent notamment Google pour chercher les informations demandées dans leurs travaux scolaires (Dumouchel et Karsenti, 2018). Ce changement fait en sorte que des compétences spécifiques doivent être développées chez les élèves du primaire et du secondaire pour chercher l'information de façon efficace. Parmi celles-ci se trouvent les compétences informationnelles qui sont présentes dans les documents d'orientation des différents ministères de l'éducation des pays industrialisés. Elles sont réitérées à la dimension 4 du continuum de la compétence numérique «Développer et mobiliser sa culture informationnelle»(Gouvernement du Québec, 2019). Parmi les suggestions de thèmes à aborder, mentionnons les moteurs de recherche, les mots-clés ainsi que les opérateurs booléens ou logiques. En effet, les stratégies de recherche d'information en ligne sont parmi les plus importantes variables à développer tant pour les enseignants que pour les élèves, et ce, pour favoriser une exploitation optimale des ressources disponibles sur Internet. Toutefois, les recherches démontrent que les enseignants du primaire et du secondaire nont pas nécessairement reçu la formation adéquate pour intégrer ces stratégies de recherche dans leur pratique pédagogique (Dumouchel et Karsenti, 2019; Karsenti et Grégoire, 2015; Simard, Karsenti et Collin, 2013). En conséquence, cela peut amener les enseignants à avoir des réserves quant à l'utilisation des technologies en salle de classe en raison de leur manque de connaissances relatives aux potentialités des TIC et de leurs usages (Karsenti et Grégoire, 2015; Larose, Grenon, Carignan et Hammami, 2010; Lawrence et Tar, 2018). Ces réserves peuvent entrainer un enseignement plus 
traditionnel qui restreint le recours aux technologies (Dumouchel et Karsenti, 2018; Lei, 2009) ou qui n'en exploite pas toutes les fonctionnalités. Pour respecter l'esprit du programme de formation et du nouveau continuum de développement de la compétence numérique (Gouvernement du Québec, 2019), les enseignants doivent agir en tant que modèle en employant et en faisant la promotion de l'utilisation des outils et des ressources d'information en ligne pour l'apprentissage de leurs élèves en les intégrant à leurs pratiques d'enseignement.

Les stratégies de recherche sont donc très importantes dans le développement des compétences informationnelles à la fois des enseignants et des élèves. Certaines recherches se sont intéressées au mode de fonctionnement des élèves du primaire et du secondaire (et non de l'enseignant) lors de la lecture sur un support papier ou à l'écran, mais celles-ci ont mis l'accent sur les stratégies de lecture utilisées et le degré de compréhension des élèves (Carignan, 2007, 2008, 2009a, 2009b, 2010a, 2010b; Carignan et Grenon, 2012; Carignan, Grenon et Caron, 2012; Lacelle, Boutin et Lebrun, 2017; Rouet, Ros, Goumi, Macedo-Rouet et Dinet, 2011; Rouet, 2012; Coiro, 2011; Salmerón, García et VidalAbarca, 2018). Dans cette optique, nous nous sommes penchés sur la façon dont les compétences informationnelles pouvaient être enseignées en milieu scolaire. Nous désirions notamment en connaitre plus sur les méthodes utilisées par les enseignants et leur niveau de connaissance des stratégies de recherche. En effet, il existe très peu de recherches à ce sujet (Chauret, 2018; Dumouchel et Karsenti, 2018, 2019).

Ainsi, peu de documents sont à la disposition des enseignants pour enseigner efficacement les compétences informationnelles en salle de classe ainsi que les stratégies de recherche associées. Par conséquent, l'objectif de cet article est d'identifier les compétences informationnelles déclarées par des enseignants du primaire et du secondaire lors d'une recherche par mots-clés sur le moteur de recherche Google. Dans la partie suivante de cet article, le concept ${ }^{1}$ de lecture à l'écran sera approfondi.

\section{Lecture à l'écran}

Lorsqu'on parle de lecture à l'écran², il peut s'agir de la lecture sur différents supports électroniques (ordinateur, tablette, téléphone, liseuse, etc.), de documents numériques ayant une structure linéaire (traitement de texte, PDF) ou de contenus Web de toute nature (textes, hyperliens, vidéos, images fixes, images animées, publicités, etc.) (définition adaptée de Lacelle et al., 2017). De plus, la lecture à l'écran est un concept qui englobe les compétences informationnelles et les stratégies de lecture à l'écran. 
Voici un schéma expliquant le concept de lecture à l'écran :

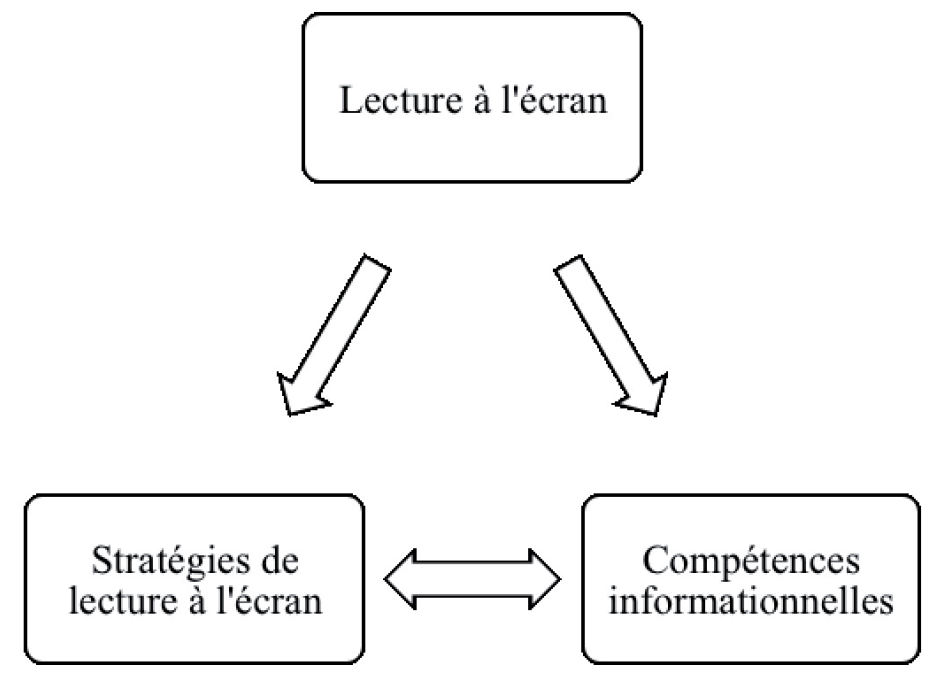

Figure 1

La lecture à l'écran ${ }^{3}$

Lors de la lecture à l'écran, les compétences informationnelles et les stratégies de lecture à l'écran sont en constante interaction. En guise d'exemple, lorsque quelqu'un utilise un moteur de recherche, il doit entrer des mots-clés (compétences informationnelles). Par la suite, selon les résultats, la personne doit lire partiellement les résultats de recherche (stratégie de lecture à l'écran). Et ainsi de suite.

À l'écran, le mode de lecture change (Baccino et Drai-Zerbib, 2015). Cependant, afin d'orienter les interventions pédagogiques des enseignants en matière de compétences informationnelles, il est nécessaire de mieux comprendre la manière dont les enseignants effectuent leur recherche sur Internet. Voilà pourquoi, dans le cadre de cet article, nous allons mettre l'accent sur les compétences informationnelles utilisées par les enseignants du primaire et du secondaire.

\section{Compétences informationnelles}

Les compétences informationnelles consistent en un ensemble d'habiletés englobant la découverte d'informations, la compréhension de l'information produite, et l'utilisation de l'information en créant de nouvelles connaissances ${ }^{4}$ (Association of College and Research Libraries, ACRL, 2015, traduction libre). Ces compétences informationnelles ont été développées depuis les années 2000 et ces habiletés ont été reconduites par le cadre de l'ACRL en 2015.

Pour être en mesure d'effectuer un traitement de l'information adéquat, le cyberlecteur doit connaitre les stratégies de recherche efficace d'informations et savoir comment les mettre en œuvre convenablement. 


\section{Stratégies de recherche}

La stratégie de recherche (searchstrategy) est une organisation structurée de termes utilisés pour chercher efficacement sur une base de données (University library, 2017). La stratégie de recherche montre comment ces termes peuvent se combiner afin de récupérer les meilleurs résultats possibles en fonction de son intention de recherche au départ. Les informations recueillies sur Internet se présentent sous divers formats (PDF, vidéos, images, texte HTML, audio, etc.), soulevant ainsi des questions relatives à leur authenticité, à leur validité et à leur fiabilité (Mottet et Gagné, 2017). Certains auteurs rapportent que les enseignants, en raison d'un certain manque en formation initiale, possèdent des compétences informationnelles limitées (Dumouchel et Karsenti, 2018; Grenon, 2007; Ntuli et Kyei-Blankson, 2016). Par conséquent, ils doivent composer avec des habiletés réduites pour les enseigner efficacement à leurs élèves.

\section{Modèles du processus de recherche d'information}

Plusieurs modèles du processus de recherche d'information ont été créés au fil des années. S'inspirant de l'ACRL (2000), Rosman, Mayer et Krampen (2015) ont développé une grille d'évaluation des stratégies de recherche constituée des compétences de la recherche d'information en psychologie pouvant ainsi être utilisée dans le milieu universitaire. Karsenti, Dumouchel et Komis (2014), quant à eux, ont proposé un modèle permettant de réfléchir aux habitudes de recherche et d'identifier les compétences informationnelles utilisées par les étudiants universitaires. Pour l'élaboration du modèle portant sur les stratégies de recherche, nous nous sommes en partie inspirés des recherches de Rosman et al. (2015), de Karsenti et al. (2014) et de l'ACRL (2000).

La structure du modèle de Karsenti et al. (2014) nous a permis d'élaborer les trois étapes du processus de recherche d'information, soit la planification de la recherche, le traitement de l'information et l'usage de l'information constituant l'organisation générale de leur modèle. La section Recherche d'information du nouveau modèle de compétences informationnelles adapté de (Chauret, 2018) est inspirée de celui de Rosman et al. (2015) et de l'ACRL (2000). Il est important de mentionner que ce modèle adapté (Chauret, 2018) se veut une suggestion d'étapes successives à employer et à enseigner, mais ne constitue pas une obligation d'étapes à utiliser de façon linéaire. En effet, il peut y avoir des allers-retours entre les différentes étapes et certaines peuvent tout simplement être omises.

\section{Étape 1 : planification de la recherche}

La première étape de la recherche d'information est la planification de la recherche. Cette étape est divisée en trois sous-étapes : définir le sujet, déterminer les stratégies adoptées et recherche d'information (voir figure 2). Tout d'abord, le cyberlecteur doit, dans sa planification, définir le sujet de sa recherche. Pour ce faire, il doit avoir un besoin informationnel, une description claire de son problème, du type de problème ainsi que la quantité d'informations nécessaires à sa résolution de problème (Brand-Gruwel, Wopereis et Vermetten, 2005). Le cyberlecteur se questionne donc sur le thème de sa recherche, le type de recherche qu'il doit effectuer, le public cible, les consignes et les différentes étapes à respecter. Ensuite, il va cibler les aspects de son sujet pour ainsi guider sa recherche (ACRL, 2000) en définissant les concepts clés et les termes. Par exemple, pour un sujet comme «l'utilisation des tablettes dans les écoles du primaire : situation en France», nous pouvons sélectionner les quatre mots-clés suivants : utilisation, tablette, primaire, France. Pour éviter d'orienter la recherche, l'emploi de termes décrivant des relations 
conditionnelles (par ex. : utilisation du mot «difficulté») ou de conséquences doit être évité. En outre, la sélection du mot-clé «France» peut éliminer les recherches à l'extérieur du pays.

Pour la deuxième sous-étape de la planification de la recherche, le cyberlecteur doit déterminer les stratégies de recherche adoptées. Pour ce faire, il doit choisir les outils technologiques les plus adaptés (Google, Google Scholar, ouvrages spécialisés, bases de données spécialisées comme ÉRIC, Érudit, PsycInfo, etc.), qu'il veut employer pour accéder à l'information recherchée. De plus, le cyberlecteur doit élaborer la démarche adoptée ou les démarches adoptées pour répondre à son besoin d'information pour en planifier, par exemple, les diverses étapes à mettre en place et déterminer comment et par quel moyen il va le faire.

La recherche d'information est la troisième sous-étape de la planification de la recherche. Pour améliorer la pertinence des résultats sur les moteurs de recherche, le cyberlecteur peut utiliser les paramètres de recherche avancée. Dans ce cas, le cyberlecteur peut, à tout moment, prendre en compte les mots-clés proposés par le moteur de recherche Google. La personne recherchant de l'information sur le web peut aussi utiliser les trois opérateurs booléens (AND/ET; OR/OU; NOT/SAUF) qui servent à combiner plusieurs termes en utilisant les synonymes ou quasi-synonymes (air OU mélodie) ou en employant l'exclusion de termes ou de contenus (musique NOT jazz) (Mottet, Morin et Gagné, 2013). Il peut également définir une tranche chronologique en utilisant des opérateurs numériques (=; $>$; <, etc.). Par exemple, il est possible de limiter les dates de parution après les années 2000 (> 2000) (Mittermeyer et Quirion, 2003). Pour restreindre la recherche, le chercheur d'information peut faire l'utilisation de limiteurs. Ceux-ci permettent de mieux définir la recherche en choisissant des années précises, une population cible, un groupe d'âge, une langue ou les types de publication recherchés (ACRL, 2013). De son côté, la troncature (*) est employée pour remplacer des lettres manquantes pour avoir accès à la racine des mots, au singulier et au pluriel, etc. (Mittermeyer et Quirion, 2003). Par exemple, le mot grecque peut être tronqué grec* comme mots-clés, ce qui permettrait de rechercher des documents contenant grec, gréco-latin, grecque, grécité, etc. Il est aussi possible de rechercher une expression exacte en la mettant entre guillemets ("compétences informationnelles») dans le moteur de recherche. Enfin, le chercheur d'information peut modifier et ajuster des termes de sa recherche en utilisant différents «mots-clés, des synonymes et des termes connexes pour [obtenir] l'information nécessaire» (ACRL, 2000, p. 9) s'il éprouve des difficultés à trouver l'information désirée.

\section{Étape 2 : traitement de l'information}

La deuxième étape est liée au traitement de l'information. Afin de parvenir à une compréhension adéquate du sujet, le cyberlecteur doit traiter les informations en les analysant en profondeur et en faisant une classification des informations selon divers critères (format, sujet, date) pour ainsi effectuer une sauvegarde des informations. Il pourrait alors retravailler ses critères en les mettant en relation avec ses connaissances antérieures et (re)structurer sa compréhension de l'ensemble du sujet (Brand-Gruwel et al., 2005). Dans la section sauvegarde de l'information, il est question de la façon dont les informations seront conservées par le cyberlecteur. Dans ce cas, l'information peut être sauvegardée sur papier, par courriel, par l'ajout d'un onglet aux favoris du navigateur ou par l'entremise d'un document électronique, par exemple. Par la suite, pour atteindre les attentes informationnelles désirées, le chercheur d'information doit faire une évaluation des documents recueillis. Pour ce faire, il peut utiliser une grille d'évaluation (Mottet, 2014) pour vérifier la validité et la pertinence des sources d'information. Cette grille est basée sur des critères tels que la fiabilité, la validité, la précision, l'exhaustivité, la disponibilité, etc. (Brand- 
Gruwel et al., 2005). En dernier, pour s'assurer de répondre aux objectifs de la recherche établis lors de la planification de la recherche (étape 1), le cyberlecteur doit synthétiser l'information en effectuant, par exemple, un résumé de l'information recueillie avec un tableau, une feuille de note, etc.

\section{Étape 3 : usage de l'information}

Afin de répondre à son intention de départ, le chercheur d'information doit, lors de la troisième étape liée à l'usage de l'information, élaborer un questionnement sur ce qu'il désire faire avec les informations recueillies (rapport, situation d'enseignement-apprentissage, présentation orale, etc.). Par la suite, il doit effectuer la synthèse des informations recueillies au cours de sa recherche pour en arriver à une transmission des connaissances. Cette synthèse peut prendre diverses formes telles qu'une affiche, un diaporama, un rapport de recherche, une carte conceptuelle, une présentation, etc. Selon la tâche à effectuer et les critères de départ, la forme finale du produit peut prendre le format d'un texte (Mottet, 2014), d'un exposé oral, d'une affiche, etc.

La figure 2 qui suit est le modèle adapté de compétences informationnelles de Chauret (2018). Comme nous l'avons déjà mentionné, ce modèle reprend celui de Karsenti et al. (2014) pour la structure en trois étapes (planification de la recherche, traitement de l'information et usage de l'information), ainsi que celui de Rosman et al. (2015) et de l'ACRL (2000) pour l'intégration de différentes stratégies de recherche dans la partie sur la recherche d'information.

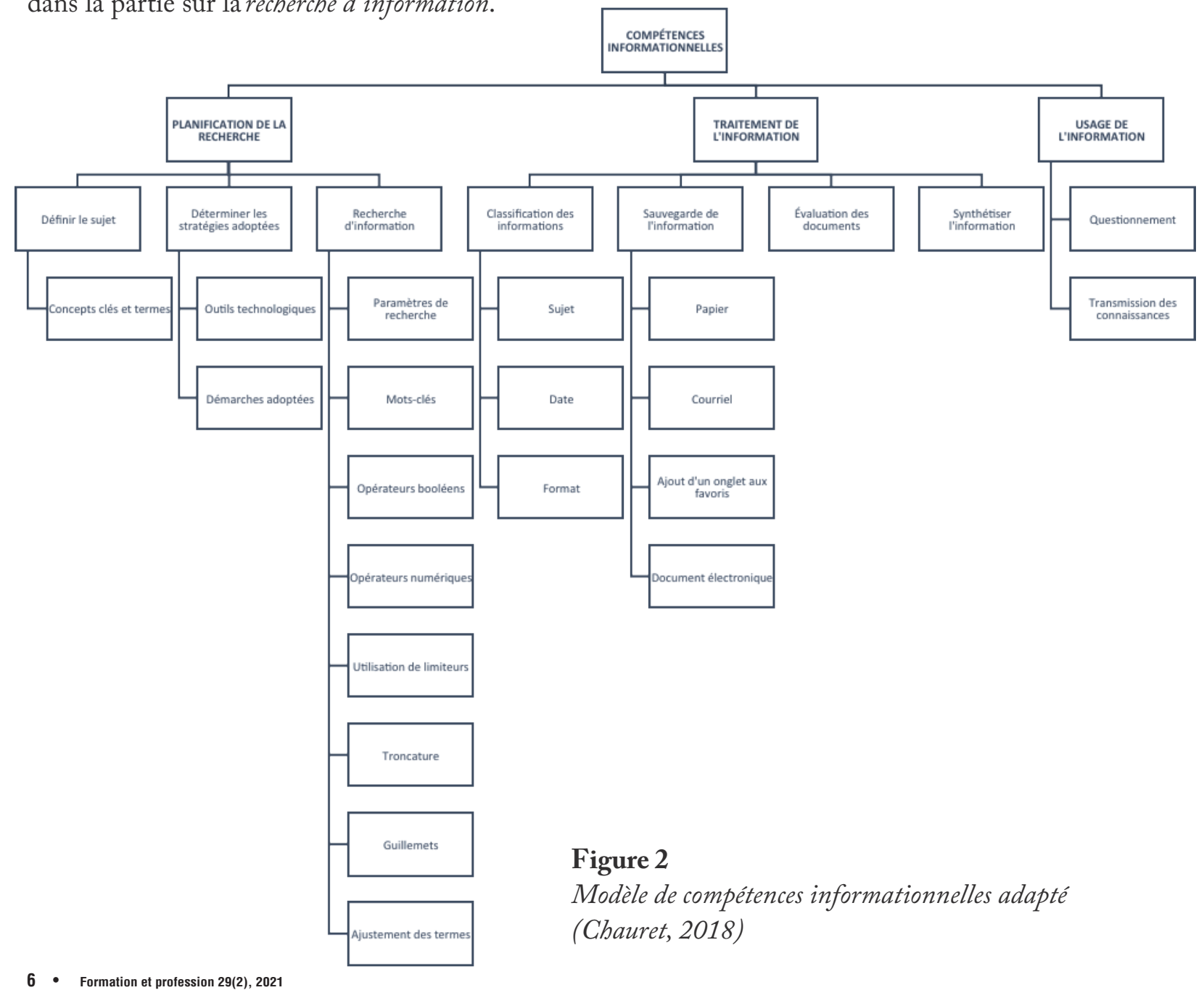




\section{Méthodologie}

Notre étude est descriptive puisque nous désirons observer «les caractéristiques d'un phénomène ou d'une population» (Fortin et Gagnon, 2016, p. 224). Elle est également qualifiée d'exploratoire, car il s'agit «[d'un] thème peu analysé et dont le chercheur ne peut établir un portrait de la situation à partir des connaissances existantes» (Gauthier, 2009, p. 171). Le recrutement des participants a été réalisé par voie électronique à partir du réseautage des chercheurs. La participation à la recherche s'est faite sur une base volontaire. Notre échantillon en est un de convenance non probabiliste (Beaud, 2009).

La recherche comporte sept enseignants dont 4 du primaire et 3 du secondaire, pour un total de sept participants. Il y avait six femmes et un homme. La moyenne d'âge est de 33,86 ans et la moyenne d'expérience en enseignement est de 9,43 ans.

\section{Outils méthodologiques}

\section{Verbalisation concurrente à la réalisation de la tâche}

Loutil méthodologique utilisé est la verbalisation concurrente à la réalisation de la tâche. Celle-ci consiste en l'explication par le participant de ses pensées et de ses actions (Fortin et Gagnon, 2016). Le but de cette verbalisation est de documenter les compétences informationnelles déclarées par les participants lors de leur recherche d'information à l'écran. Celle-ci a été enregistrée à l'aide du logiciel Virage. Ce logiciel permet d'enregistrer tous les mouvements des participants à l'écran ainsi que leur verbalisation en temps réel.

\section{Entrevue libre}

L'entrevue libre a été utilisée pour recueillir « de l'information en vue de comprendre la signification d'un évènement ou d'un phénomène vécu par les participants, conformément à l'intention du chercheur» (Fortin et Gagnon, 2016, p. 319). L'entrevue libre avait lieu en même temps que la verbalisation concurrente à la réalisation de la tâche.

\section{Analyse des données}

Pour décrire et analyser les données provenant de la verbalisation concurrente à la réalisation de la tâche et de l'entrevue libre, nous avons opté pour l'analyse de contenu (Bardin, 2013). D'une part, les verbatims ont été transcrits. D'autre part, le codage des données a été fait selon une grille d'analyse des stratégies de recherche préalablement établie. Enfin, un dénombrement de la fréquence des stratégies de recherche a été effectué afin de déterminer les stratégies de recherche utilisées par les enseignants lors d'une recherche par mots-clés sur le moteur de recherche Google. Pour établir la validité des analyses, un accord interjuges a été effectué. Deux verbatims sur sept ont été codés et contrecodés en entier : il s'agit du premier verbatim ainsi que celui qui soulevait le plus de problèmes. Il s'agit de $29 \%$ du corpus, ce qui correspond à un nombre plus élevé que ce qui est minimalement recommandé, soit $10 \%$ (Bourgeois, 2016). Avant la discussion, l'accord interjuges était de $80 \%$. Après celle-ci, l'accord interjuges s'est élevé à $100 \%$. 


\section{Déroulement de l'expérimentation}

L'expérimentation s'est réalisée au LORIT, le laboratoire du Centre de recherche LICEF de l'Université TÉLUQ au campus de Montréal. Les participants $(\mathrm{n}=7)$ devaient effectuer, sur le moteur de recherche Google, une recherche par mots-clés sur un sujet libre. En s'imaginant être devant leurs élèves, ils devaient verbaliser à voix haute leur cheminement métacognitif lors de leur recherche et expliquer pourquoi ils sélectionnaient ou non les documents à l'écran. Ils devaient, sans limites de temps, sélectionner au minimum deux documents numériques représentatifs de leur intention de lecture/recherche de départ et en expliquer les raisons. L'assistante de recherche était cachée dans une autre pièce et pouvait suivre sur un autre écran tous les mouvements des participants et leur verbalisation pendant la réalisation de la tâche demandée. Ce contexte a permis aux participants de se sentir plus à l'aise lors de la réalisation de la recherche par mots-clés et de se sentir moins observés. Ces possibilités offertes par la configuration spatiale du laboratoire (salle isolée et proximité avec le participant sans occasionner un sentiment d'être observé constamment) ont favorisé la réalisation de l'étude dans un contexte plus authentique qui représentait ce que ferait un enseignant lors d'une recherche typique en salle de classe.

\section{Résultats et interprétations}

Dans cette section, les stratégies de recherche déclarées par les participants lors de la recherche par mots-clés à l'écran seront décrites. Lors de la verbalisation concurrente à la réalisation de la tâche, les sept sujets ont déclaré utiliser, en moyenne, 9,43 types de stratégies de recherche $(s=2,51)$ sur les 20 qu'il est possible d'employer selon le modèle adapté de Chauret (2018). Au total, 283 stratégies de recherche ont été déclarées par les participants. De façon générale, la stratégie de recherche d'information a été la plus déclarée, soit à 112 reprises. Elle fait partie de la première étape du modèle de compétences informationnelles. La deuxième stratégie la plus utilisée est l'ajustement des termes de la recherche, déclarée à 29 reprises. Elle fait également partie de la première étape du modèle, soit la planification de la recherche.

Nous allons maintenant décrire les 21 stratégies de recherche déclarées par les sujets (voir tableaux 1,2 et 3) en nous basant sur la verbalisation concurrente à la réalisation de la tâche des participants.

\section{Planification de la recherche (étape 1)}

Comme nous venons de le mentionner, la recherche d'information a été employée par tous les participants. Elle a été déclarée 112 fois au total. Nous en faisions la recension lorsque le participant activait un hyperlien puisque cette activation correspond à une recherche d'information. Donc, nous l'avons recensée lorsque le participant sélectionnait l'hyperlien afin d'obtenir de nouvelles informations sur des images : «Bon là je viens de voir un autre lien Pinterest $2^{\mathrm{e}}$ cycle, les machines simples, je vais aller voir plus pour des images sciences, $2^{\mathrm{e}}$ cycle, en général» (P1).

La recherche d'information $(\mathrm{n}=112)$ est souvent en cooccurrence ${ }^{5}$ avec la stratégie réalisation d'une double recherche $(\mathrm{n}=15)^{6}$, déclarée par quatre participants. Par exemple, un participant cliquait sur l'hyperlien pour lire les informations s'y trouvant en plus de l'ouvrir dans un nouvel onglet : «Donc, j’irais directement sur leur site, dans leur contenu Web. Vidéo. Exclusivité Web et épisodes»(P7). 
Les participants ont employé la stratégie réalisation d'une double recherche dans deux différents contextes, par exemple en utilisant le moteur de recherche Google image afin de visualiser le personnage mentionné dans le contenu de l'hyperlien ou encore en ouvrant un nouvel onglet avec les hyperliens qui l'intéressaient pour ainsi poursuivre sa recherche actuelle. Cette dernière action est plus complexe puisque le cyberlecteur se retrouve avec plusieurs hyperliens ouverts simultanément, ce qui pourrait amener une surcharge cognitive (Sweller, 1994) en raison de la gestion plus difficile de la recherche.

Six participants ont, au cours de leur recherche, fait l'ajustement des termes de recherche $(\mathrm{n}=29)$. L'utilisation de cette stratégie leur a permis d'obtenir de plus amples informations sur le sujet recherché ou d'effectuer une comparaison entre les informations obtenues par les mots-clés sélectionnés préalablement : «Je vais changer ma recherche, qu'est-ce qu'on mettrait? Si on fait juste changer la «vie» par non je ne pense pas, je vais essayer. «Vivre au Moyen-Âge» [ajustement des termes] au lieu de la «vie au Moyen-Âge» ( $\mathrm{P} 3)$. La forte utilisation de cette stratégie est due au fait que les cyberlecteurs doivent ajuster les mots-clés lors de leur recherche s'ils n'obtiennent pas les résultats désirés ou que les hyperliens proposés par Google ont tous été vus.

L'utilisation des outils technologiques $(\mathrm{n}=7)$, déclarée par quatre répondants sur sept, a été employée lorsque les participants désiraient, par exemple, se faire une image sur ce qu'ils venaient de lire : «j'irais dans Google image». Cette stratégie a été peu utilisée puisque nous avons restreint la recherche à l'utilisation du moteur de recherche Google uniquement.

Cinq participants ont identifié les concepts clés et thèmes $(\mathrm{n}=6)$ de leur recherche en cooccurrence avec la stratégie identification du public cible $(\mathrm{n}=1)$, déclarée uniquement par un participant. Cette stratégie a été ajoutée au modèle adapté révisé (voir figure 3). Par exemple, le participant va définir le sujet de sa recherche tout en déterminant le niveau scolaire pour lequel il désire obtenir des informations : «les machines simples [thème], deuxième cycle du primaire [identification du public cible]» (P1). Cette faible utilisation de la stratégie identification du public cible peut s'expliquer par le fait que les enseignants nont pas éprouvé le besoin de verbaliser au sujet des destinataires au commencement de leur recherche d'information ; c'était implicite dans leur tête.

Deux des participants ont planifié la démarche adoptée $(\mathrm{n}=3)$ en préparant au préalable les motsclés qu'ils allaient utiliser dans leur recherche : «Si ça ne fonctionne pas, je vais aller dans littérature jeunesse» (P5). Toutefois, l'expérimentation ne nous a pas permis de définir le type de procédé utilisé par les participants. En effet, nous désirions recueillir des données quant à la manière dont les participants élaboraient la planification de leur recherche, c'est-à-dire si elle était bien planifiée, semi-planifiée, etc.

Deux participants ont employé la stratégie regarder la possibilité de faire une recherche dans une autre langue $(\mathrm{n}=3)$ qui est une stratégie que nous avons ajoutée au modèle adapté révisé (figure 3). Lors de leur recherche, certains participants, qui étaient à l'aise avec la langue anglaise, ont effectué une recherche en utilisant des mots-clés en anglais ou en allant sur des sites Internet de langue anglaise. Par exemple, ce participant utilise un site en anglais pour conclure sa recherche : «j'ai un site en anglais. Polyester fabric. Ouin, je vais y aller après, peut-être» (P4).

Exprimés par un seul participant, les paramètres de recherche $(\mathrm{n}=2)$ ont été verbalisés lorsque le participant a utilisé les paramètres avancés du moteur de recherche Google afin de raffiner sa recherche : 
«donc je vais aller dans «mes paramètres outils », tous les pays, je vais sélectionner juste Canada, ça va m'aider rapidement à faire le tri» (P7). Ce faible dénombrement est dû au fait que nous avons restreint l'outil technologique utilisé au moteur de recherche Google.

La stratégie utilisation du moteur de recherche de l'hyperlien $(\mathrm{n}=2)$, stratégie que nous avons ajoutée au modèle adapté révisé (figure 3), est exprimée par un seul participant sur sept. Un participant a utilisé l'onglet «recherche» du site Internet pour avoir accès aux informations sur un produit plus rapidement. Comme mentionné dans l'extrait, le participant écrit le mot du produit qu'il recherche dans l'onglet «recherche» : «Ok, bon si je viens ici puis je fais nylon, ok nylon. Qu'est-ce que je vais trouver» (P4).

L'utilisation de limiteurs $(\mathrm{n}=1)$ a été utilisée une seule fois par un participant. Ce dernier a limité sa recherche en utilisant le menu du site Internet puisqu'il considérait qu'il avait trop d'informations à trier dans l'hyperlien sélectionné.

De nombreuses stratégies provenant du modèle adapté (figure 2) nont pas ou ont très peu été déclarées par les participants lors de la tâche. Par exemple, aucun des participants n'a utilisé d'opérateur booléen, d'opérateurs numériques, de troncature ou de guillemets. Leur inutilisation laisse entrevoir une méconnaissance de ceux-ci ou de leur potentiel par les participants.

Voici un résumé des stratégies de recherche déclarées par les participants lors de la première étape de la recherche, soit la planification de la recherche.

\section{Tableau 1}

Planification de la recherche (étape 1)

Planification de la recherche (étape 1): stratégies de recherche déclarées

Recherche d'information
Ajustement des termes
Concepts clés et termes
Réalisation d'une double recherche
Outils technologiques
Délimitation du sujet
Démarche(s) adoptée(s)
Recherche dans une autre langue
Paramètres de recherche
Utilisation du moteur de recherche de I'hyperlien
Identification du public cible
Utilisation de limiteurs
Opérateurs booléens
Troncature
Guillemets
Opérateurs numériques

Fréquence

Nombre de sujets

|

$\begin{array}{ll}112 & 7 / 7 \\ 29 & 6 / 7 \\ 22 & 6 / 7 \\ 15 & 4 / 7 \\ 7 & 4 / 7 \\ 6 & 5 / 7 \\ 3 & 2 / 7 \\ 3 & 2 / 7 \\ 2 & 1 / 7 \\ 2 & 1 / 7 \\ 1 & 1 / 7 \\ 1 & 1 / 7 \\ 0 & 0 / 7 \\ 0 & 0 / 7 \\ 0 & 0 / 7 \\ 0 & 0 / 7\end{array}$

Note : les mots en gras sont les nouvelles stratégies de recherche recensées et intégrées dans la version révisée du modèle adapté de Chauret (2018) à la figure 3. 


\section{Traitement de l'information (étape 2)}

L'évaluation des documents $(\mathrm{n}=27)$, déclarée par six participants sur sept, a été recensée lorsqu'un participant jugeait le contenu d'un site internet. Par exemple, lorsqu'il disait : «ça me dit qu'au niveau de la fiabilité peut-être qu'il faudrait colliger certaines informations» (P2). Sa forte utilisation s'explique par le fait que les cyberlecteurs savent qu'ils doivent porter un jugement critique sur l'auteur, la pertinence et l'exactitude des informations, la provenance, etc., des hyperliens sélectionnés.

Cela dit, les informations recueillies lors de la recherche par les participants ont été sauvegardées de diverses façons. En effet, quatre participants (sur sept) ont écrit la source sur papier $(n=11)$. Nous l'avons recensé lorsque les participants le mentionnaient : «Je vais me le noter, mais je vais essayer de trouver mieux» (P3). Un participant a fait l'utilisation d'un service de stockage $(\mathrm{n}=1)$, plus particulièrement Dropbox. En effet, le participant 5, à la fin de sa recherche, a transféré les articles scientifiques trouvés dans son Dropbox. Un participant a fait, à huit reprises, le téléchargement de documents sur le bureau de l'ordinateur. Nous l'avons observé lorsqu'un participant disait, par exemple : «Je vais le télécharger, je m’intéresse à leur méthodologie»(P5). Aucun enseignant n'a envoyé les sources trouvées par voie électronique. De plus, comme ils n'utilisaient pas leur ordinateur personnel ou professionnel, il était inutile d'ajouter un onglet dans les favoris du navigateur.

La stratégie synthétiser l'information $(\mathrm{n}=5)$, déclarée par trois participants sur sept, a été utilisée, par exemple, lorsqu'un participant vérifiait les informations recueillies pour s'assurer qu'il possédait toutes les informations désirées : «Donc, on me rassure j’ai déjà mes deux liens avec mes deux baladodiffusions et j'ai Alt qui est plus jeunesse» (P7). Pratiquement la moitié des participants ont synthétisél'information recueillie verbalement. Il est possible de penser que les participants s'assuraient de répondre aux critères et aux buts spécifiques de la recherche qu'ils avaient préalablement ciblés lors de la planification de leur recherche.

La stratégie de classification des informations recueillies $(n=4)$, déclarée par deux participants sur sept, est cooccurrente à la stratégie classer par sujet $(\mathrm{n}=1)$ qui est utilisée seulement par un participant, dans l'extrait suivant: "Je vais écouter cela lui, je vais choisir mon meilleur et effacer l'autre» (P7). Donc, le sujet sélectionne l'hyperlien le plus pertinent et le mieux conçu afin de répondre à son objectif de recherche. La faible utilisation de la stratégie classification des informations peut s'expliquer par le type de tâche demandée. En effet, la tâche n'exigeait pas que les participants effectuent un classement; ils feuilletaient plutôt les informations sur Internet portant sur le sujet sélectionné sans devoir construire de matériel pour une situation d'enseignement et d'apprentissage. 
Voici un tableau récapitulatif des stratégies de recherche déclarées à la deuxième étape, soit le traitement de l'information.

\section{Tableau 2}

Traitement de l'information (étape 2)

\begin{tabular}{|c|c|c|}
\hline $\begin{array}{l}\text { Traitement de l'information (étape 2): } \\
\text { stratégies de recherche déclarées }\end{array}$ & Fréquence & Nombre de sujets \\
\hline Évaluation des documents & 27 & $6 / 7$ \\
\hline Papier & 11 & $4 / 7$ \\
\hline Synthétiser l'information & 5 & $3 / 7$ \\
\hline Classification des informations & 4 & $2 / 7$ \\
\hline Téléchargement de documents & 8 & $1 / 7$ \\
\hline Sujet & 1 & $1 / 7$ \\
\hline Utilisation d'un service de stockage & 1 & $1 / 7$ \\
\hline Date & 0 & $0 / 7$ \\
\hline Format & 0 & $0 / 7$ \\
\hline Courriel & 0 & $0 / 7$ \\
\hline Ajout d'un onglet aux favoris & 0 & $0 / 7$ \\
\hline Document électronique & 0 & $0 / 7$ \\
\hline
\end{tabular}

\section{Usage de l'information (étape 3)}

Six participants ont employé la stratégie transmission des connaissances $(\mathrm{n}=26)$. Elle est observable lorsque le participant mentionne : «j'aime bien ça utiliser des livres pour expliquer ce que j’enseigne et ces livres-là ensuite je les mets en lecture libre. C'est super populaire»(P1). Donc, nous pouvons entrevoir ce que le participant désire faire avec le matériel.

Un seul participant a employé le questionnement $(\mathrm{n}=1)$. Ce participant s'est questionné à savoir s'il avait toute l'information requise pour répondre à son objectif et à son questionnement de départ : «je m'arrêterais de fouiller dans les sites parce que j'ai quand même plusieurs pays» (P2). 


\section{Tableau 3}

Usage de l'information (étape 3)

Usage de l'information (étape 3 )

Fréquence

Nombre de sujets

Stratégies de recherche déclarées

\begin{tabular}{r|cc} 
Transmission des connaissances & 26 & $6 / 7$ \\
Questionnement & 1 & $1 / 7$
\end{tabular}

Dans la prochaine section, il sera question des raisons expliquant les ajouts au modèle de compétences informationnelles adapté de Chauret (2018).

\section{Modifications apportées au modèle de compétences informationnelles}

Plusieurs modifications ont été apportées au modèle adapté de Chauret (2018) lors de l'analyse des données. En tout, six stratégies ont été ajoutées, soit quatre dans la première étape (planification de la recherche) et deux dans la deuxième étape (traitement de l'information). Celles-ci ont été mises en majuscules, en gras et en couleur bleue dans la figure 3. Tout d'abord, nous avons supprimé/rassemblé la stratégie de recherche déterminer les stratégies adoptées de la première étape puisqu'il y avait une certaine redondance avec la stratégie démarches adoptées. Nous avons renommé cette partie démarche(s) adaptée(s) et stratégies utilisées.

Par la suite, nous avons supprimé/rassemblé la stratégie de recherche mots-clés de la deuxième étape, puisqu'il y avait une confusion - et une redondance - avec la stratégie définir les concepts clés et termes de la recherche. Par conséquent, lorsqu'un participant définissait ses mots-clés, nous l'avons codé comme définir les concepts clés/termes dans la première étape, lors de la planification de la recherche. Nous avons renommé cette partie concepts clés/termes (mots-clés).

Nous avons ajouté la stratégie identification de son public cible puisque certains participants le faisaient.

Dans la partie «recherche d'information», de l'étape 1, il y a eu l'ajout de la stratégie réalisation d'une double recherche dès l'instant où nos participants ont ouvert un nouvel onglet pour aller recueillir de plus amples informations sur le sujet dans l'hyperlien. Par ailleurs, dans cette même section, nous avons ajouté l'utilisation du moteur de recherche de l'hyperlien puisque le moteur de recherche présent sur la page active a été utilisé par nos participants pour atteindre plus rapidement l'information désirée. Nous avons également ajouté la stratégie recherche dans une autre langue, puisque plusieurs des participants ont changé la langue de leur recherche au courant de celle-ci.

Au cours de la deuxième étape (traitement de l'information), dans la section classification des informations recueillies, une stratégie de recherche a été ajoutée dans la sauvegarde de l'information. En effet, nous avons ajouté le téléchargement de documents puisque l'un de nos participants téléchargeait les documents 
numériques (PDF) directement sur le bureau de l'ordinateur et nous avons ajouté utilisation d'un service de stockage puisqu'un participant a utilisé le sien.

Ce modèle a pris en compte les différentes stratégies que le cyberlecteur peut réaliser lors de sa recherche d'information, de l'utilisation de l'information et de la transmission de celle-ci. Le modèle adapté et révisé proposé (voir figure 3) vise, d'une part, à mieux refléter les stratégies de recherche de sept enseignants du primaire et du secondaire. Ce modèle permet aussi de mieux déterminer les différentes compétences qui composent l'ensemble des compétences informationnelles actuelles. D'autre part, ce modèle permet aussi d'actualiser le processus de recherche d'information tel qu'il est présenté dans la majorité des modèles de recherche d'information.

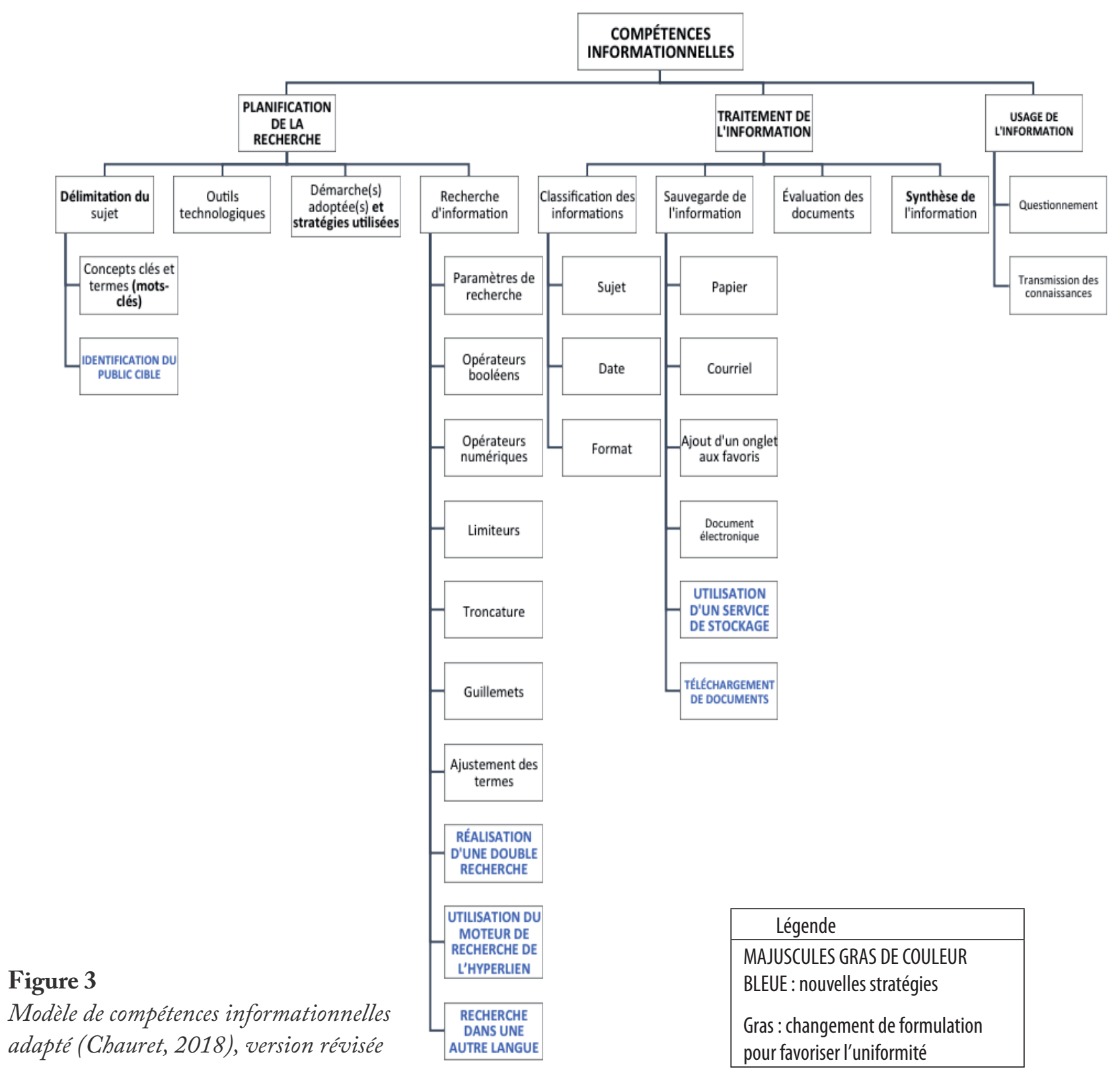




\section{Discussion, limites et conclusion}

Cette étude aura permis de documenter les compétences informationnelles déclarées par des enseignants du primaire et du secondaire lors de leur recherche par mots-clés sur le moteur de recherche Google. Nos résultats, ainsi que la version révisée du modèle de compétence informationnelle, pourront guider les formateurs universitaires dans la planification des activités de formation en lien avec l'enseignement des compétences informationnelles dans les cours de formation initiale à l'enseignement et en formation continue. Le développement de l'outil Google, qui permet maintenant d'effectuer la troncature ainsi que de nouvelles fonctions, favorise l'ouverture à de nouvelles possibilités.

La grande majorité des enseignants ont été formés avant ces transformations et ces ajouts relatifs aux différentes fonctionnalités. Il est donc normal que ceux-ci ne les utilisent pas puisqu'ils n'ont pas nécessairement reçu ce genre de formation. Ceci pourrait expliquer pourquoi nos participants n'ont pas, par exemple, fait l'utilisation de certaines stratégies de recherche comme les opérateurs booléens, les limiteurs ou la troncature.

Nous pouvons cependant nous questionner à savoir si les enseignants savent modéliser adéquatement la recherche d'information avec leurs élèves. De plus, nous pouvons nous interroger sur leurs compétences personnelles à effectuer une recherche. Par conséquent, à l'instar de Dumouchel et Karsenti (2018), nous pensons qu'offrir une meilleure formation initiale ou continue permettrait certainement aux enseignants de familiariser leurs élèves aux différentes stratégies et techniques de recherche d'information efficaces leur permettant d'exploiter les possibilités multiples qu'Internet peut leur offrir (Dumouchel et Karsenti, 2019; Laxman, 2009; Simard, et al., 2013).

Cette recherche comporte plusieurs limites. Le nombre restreint de participants représente une grande limite à la recherche. En effet, en raison du budget limité, il a été impossible d'offrir une compensation financière pour le déplacement des participants pour la participation à l'expérimentation. De plus, le nombre restreint de participants rend nos résultats non généralisables. Par conséquent, il serait pertinent de répliquer la recherche avec un plus grand nombre de participants en effectuant l'expérimentation à distance puisque plusieurs sont maintenant à l'aise avec la formation à distance et le partage d'écran rendus possibles par les outils de visioconférence. En outre, en ce qui concerne la tâche demandée, cette dernière demandait un grand effort métacognitif aux participants puisqu'ils devaient déclarer les stratégies qu'ils utilisaient. Il est donc possible que certains participants n'aient pas nommé tout ce qui se passait dans leur tête, nous limitant ainsi aux stratégies déclarées verbalement.

À moyen terme, l'objectif de cette recherche serait de déterminer les stratégies de lecture à enseigner aux élèves lors de la lecture à l'écran, et ce, de façon explicite, afin qu'ils puissent devenir des cyberlecteurs efficaces. Pour y arriver, les enseignants devront recevoir des formations adéquates non seulement sur les compétences informationnelles, mais également sur les stratégies de lecture à l'écran pour être en mesure de les enseigner par la suite de façon efficace. Pour ce faire, les enseignants devront avoir en main du matériel didactique basé sur les écrits scientifiques pour leur permettre de les enseigner adéquatement. 


\section{Notes}

1 Nous avons choisi d'utiliser la conception d'un hyperlien au lieu de perception puisque la conception d'un hyperlien est la manière dont une personne envisage l'hyperlien. Donc, comment elle le juge, comment elle le conçoit dans sa tête.

2 Nous avons opté pour le terme «lecture à l'écran» et non «lecture numérique» ou «lecture en ligne», car il est plus englobant. En effet, il est possible, par exemple, de consulter un document en format PDF ou dans un logiciel de traitement de texte sans avoir accès à Internet.

3 Ce schéma a été créé par les auteurs pour conceptualiser la lecture à l'écran.

4 «Information literacy is the set of integrated abilities encompassing the reflective discovery of information, the understanding of how information is produced and valued, and the use of information in creating new knowledge and participating ethically in communities of learning.» (ACRL, 2015, p. 8).

5 Ceci signifie que les deux stratégies de recherche sont utilisées de façon simultanée.

6 Veuillez noter que six nouvelles stratégies de recherche ont été ajoutées dans le modèle de compétences informationnelles adapté et révisé de Chauret (2018) (voir figure 3). Ces stratégies sont en gras et en majuscules dans le modèle amélioré.

\section{Références}

Association of College and Research Libraries (ACRL). (2000). Information literacy competency standards for higher education. Chicago, IL : ALA.

Association of College and Research Libraries. (2013). Information literacy competency standards for nursing. $\underline{\text { http://crln.acrl.org/content/75/1/34.full.pdf }}$

Association of College and Research Libraries (ACRL). (2015). Framework for Information literacy for higher education. Chicago, IL : ALA. http://www.ala.org/acrl/sites/ala.org.acrl/files/content/issues/infolit/framework1.pdf

Baccino, T. et Drai-Zerbib, V. (2015). La lecture numérique. Presses universitaires de Grenoble.

Bardin, L. (2013). L'analyse de contenu. Presses Université de France.

Beaud, J.-P. (2009). L'échantillonnage. Dans B. Gauthier, Recherche sociale. De la problématique à la collecte des données (éd. 5 , p.251-284). Presses de l'Université du Québec.

Bourgeois, L. (2016). Assurer la rigueur scientifique de la recherche-action. Dans I. Carignan, M.-C. Beaudry et Larose, F. (dir.), La recherche-action et la recherche-développement au service de la littératie (p. 6-20). Éditions de l'Université de Sherbrooke (ÉDUS).

Brand-Gruwel, S., Wopereis, I. et Vermetten, Y. (2005). Information problem solving by experts and novices: Analysis of a complex cognitive skill. Computers in Human Behavior, 21, 487-508.

Carignan, I. (2007). Étude des relations entre les formes de documents, les stratégies de lecture et la compréhension chez des élèves de $3^{\mathrm{e}}$ secondaire [Thèse de doctorat inédite, Université de Montréal]. Papyrus.

https://papyrus.bib.umontreal.ca/xmlui/handle/1866/18269

Carignan, I. (2008). Les stratégies de lecture efficaces déclarées à l'écran et sur papier par des élèves de $3^{\mathrm{e}}$ secondaire. Intercompreensão. Revista de Didáctica das Linguas, 14, 267-293.

Carignan, I. (2009a). La mobilisation de stratégies de lecture sur trois formes de documents en $3^{\mathrm{e}}$ secondaire. Nouveaux cahiers de la recherche en éducation, 12(2), 161-178. http://www.erudit.org/revue/ncre/2009/v12/n2/1017465ar.pdf

Carignan, I. (2009b). La fréquence d'utilisation des stratégies de lecture selon deux formes de documents à l'écran chez des élèves de $3^{\mathrm{e}}$ secondaire (14-15 ans). Étude de linguistique appliquée 153, 55-66. https://r-libre.teluq.ca/830/

Carignan, I. (2010a). Portraits de «compreneurs » à l'écran et sur papier. Vivre le primaire, 23(2), 26-27.

Carignan, I. (2010b). Quelles stratégies de lecture devrait-on enseigner à l'écran? Québec français, 159, 74-77. 
Carignan, I. et Grenon, V. (2012). Le degré de compréhension d'élèves de $3^{\mathrm{e}}$ secondaire sur trois formes de documents de type argumentatif. Revue canadienne de l'éducation, 35(1), 36-55. https://r-libre.teluq.ca/829/

Carignan, I., Grenon, V. et Caron, A. (2012). Les facteurs favorisant l'activation et la non-activation des hyperliens en 6e année du primaire. Dans M. Lebrun, N. Lacelle, et J.-F. Boutin (dir.), La littératie médiatique multimodale: de nouvelles approches en lecture-écriture à l'école et hors de l'école (p. 77-91). Presses de l'Université du Québec.

Chauret, M. (2018). Comprendre la lecture à l'écran : la recherche par mots-clés et l'activation d'byperliens chez des enseignants du primaire et du secondaire [Mémoire de maitrise, Université de Sherbrooke]. Savoirs UdeS. https://savoirs.usherbrooke.ca/bitstream/handle/11143/12610/Chauret Melodie MA 2018.pdf?sequence=3\&isAllowed=y

Coiro, J. (2011). Talking about reading as thinking: Modeling the hidden complexities of online reading comprehension. Theory Into Practice, 50(2), 107-115. https://doi.org/10.1080/00405841.2011.558435

Dumouchel, G. et Karsenti, T. (2019). Comment les futurs enseignants du Québec évaluent l'information trouvée sur le Web : une étude des pratiques déclarées et effectives. Formation et profession, 27(2) 74-87. http://dx.doi.org/10.18162/fp.2019.449

Dumouchel, G. et Karsenti, T. (2018). Comment les futurs enseignants sont-ils formés aux compétences informationnelles et comment prévoient-ils les enseigner? Une étude exploratoire menée au Québec (Canada). Revue internationale des technologies en pédagogie universitairel International Journal of Technologies in Higher Education, 15(2), 32-46.

Fortin, M.-F. et Gagnon, J. (2016). Fondements et étapes du processus de recherche (3éd.). Chenelière Éducation.

Gauthier, B. (2009). La structure de la preuve. Dans B. Gauthier, Recherche sociale. De la problématique à la collecte des données (p. 169-198). Québec: Presses de l'Université du Québec.

Gouvernement du Québec. (2019). Continuum de développement de la compétence numérique. Cadre de référence de la compétence numérique. Québec, Canada : Ministère de l'Éducation et de l'Enseignement supérieur. http://www.education.gouv.qc.ca/fileadmin/site web/documents/ministere/continuum-cadre-reference-num.pdf

Grenon, V. (2007). Impact de la formation en milieu de pratique sur les stagiaires quant au développement de leur niveau d'alphabétisation informatique, de leur sentiment d'auto-efficacité et de leurs attitudes de stress et d'utilité perçue au regard des $T I C$ [Thèse de doctorat, Université de Sherbrooke], Savoirs UdeS. https://savoirs.usherbrooke.ca/handle/11143/934

Karsenti, T., Dumouchel, G. et Komis, V. (2014). Les compétences informationnelles des étudiants à l'heure du Web 2.0 : proposition d'un modèle pour baliser les formations. Documentation et bibliothèques, 60(1), 20-30.

Karsenti, T. et Grégoire, P. (2015). Professionnalisation et développement professionnel des enseignants dans un contexte d'intégration des TICE : le cas du Québec. Distances et médiations des savoirs, (11). https://journals.openedition.org/ dms/

Lacelle, N., Boutin, J.-F. et Lebrun, M. (2017). La littératie médiatique multimodale appliquée en contexte numérique - LMM@ : Outils conceptuels et didactiques.PUQ.https://doi.org/10.2307/j.ctt1z27hcs

Larose F., Grenon V., Carignan I. et Hammami A. (2010). Les TIC en enseignement des langues au Québec : objet obscur d'un désir prescrit? Québec français, 159, 71-72.

Lawrence, J. E. et Tar, U. A. (2018). Factors that influence teachers' adoption and integration of ICT in teaching/learning process. Educational Media International, 55(1), 79-105. https://doi.org/10.1080/09523987.2018.1439712

Laxman, K. (2009). A baseline study on the Internet information search proficiencies of polytechnic students in Singapore. International Journal of Education and Development using ICT, 5(3), 115-130.

Lei, J. (2009). Digital natives as preservice teachers: What technology preparation is needed? Journal of Computing in Teacher Education, 25(3), 87-97.

Mittermeyer, D. et Quirion, D. (2003). Étude sur les connaissances en recherche documentaire des étudiants entrant au $1{ }^{\text {er }}$ cycle dans les universités québécoises. CREPUQ.

Mottet, M. (2014). Faire une recherche, ça s'apprend. http://www.faireunerecherche.fse.ulaval.ca

Mottet, M., Morin, E. et Gagné, J.-C. (2013). Faire une recherche d'information : des habiletés essentielles à développer. Formation et profession, 21(1), 68-70. 
Mottet, M. et Gagné, J.-C. (2017). Former à l’enseignement des compétences informationnelles en classe de français au Québec. Dans S. Rouissi, L. Portes et A. Stulic (dir.), Dispositifs numériques pour l'enseignement à l'université (p. 145155). L'Harmattan.

Ntuli, E. et Kyei-Blankson, L. (2016). Improving K-12 online learning: Information literacy skills for teacher candidates. International Journal of Information and Communication Technology Education, 12(3), 38-50.

Rouet, J.-F., Ros, C., Goumi, A., Macedo-Rouet, M. et Dinet, J. (2011). The influence of surface and deep cues on primary and secondary school students' assessment of relevance in Web menus. Learning and Instruction, 21(2), 205-219. https://doi.org/10.1016/j.learninstruc.2010.02.007

Rouet, J.-F. (2012). Ce que l'usage d'internet nous apprend sur la lecture et son apprentissage. Le français aujourd'bui, 3(178), 55-64.

Rosman, T., Mayer, A.-K. et Krampen, G. (2015). Measuring psychology students' information-seeking skills in a situational judgment test format: construction and validation of the PIKE-P Test. European Journal of Psychological Assessment, 2(3), 220-229.

Salmerón, L., García, A. et Vidal-Abarca, E. (2018). WebLEC: A test to assess adolescents' Internet reading literacy skills. Psicothema, 30(4), 388-394.

Simard, S., Karsenti, T. et Collin, S. (2013). Proposition d'un nouveau cadre de compétences informationnelles pour la formation initiale des enseignants : une invitation à la réflexion et à la recherche. Formation et profession, 21(3), 109-112.

Sweller, J. (1994). Cognitive load theory, learning difficulty, and instructional design. Learning Instruction, 4(4), $295-312$.

The university library (2017). Developing your search strategy. https://library.leeds.ac.uk/researcher-literature-search-strategy

Cet article est conforme à la nouvelle orthographe.

\section{Pour citer cet article}

Chauret, M., Carignan, I., Grenon, V., Collin, S. (2021). Les compétences informationnelles d'enseignants du primaire et du secondaire lors d'une recherche par mots-clés sur un moteur de recherche. Formation et profession, 29(2), 1-19. http://dx.doi.org/10.18162/fp.2021.567 